\title{
Successful Treatment for Hepatic Encephalopathy Aggravated by Portal Vein Thrombosis with Balloon-Occluded Retrograde Transvenous Obliteration
}

\author{
Naotaka Hashimoto ${ }^{a}$ Tomohiko Akahoshi ${ }^{a}$ Tetsuya Shoji ${ }^{b}$ \\ Morimasa Tomikawa ${ }^{a}$ Norifumi Tsutsumi ${ }^{a}$ Tomoharu Yoshizumi ${ }^{\mathrm{a}}$ \\ Akinobu Taketomi ${ }^{\mathrm{a}}$ Ken Shirabe ${ }^{\mathrm{a}}$ Yoshihiko Maehara $^{\mathrm{a}}$ \\ aDepartment of Surgery and Science, Graduate School of Medical Science, Kyushu \\ University, and bepartment of Surgery, Kaizuka Hospital, Fukuoka, Japan
}

\section{Key Words}

Hepatic encephalopathy - Portal vein thrombosis - Balloon-occluded retrograde transvenous obliteration

\begin{abstract}
This report presents the case of a 78-year-old female with hepatic encephalopathy due to an inferior mesenteric venous-inferior vena cava shunt. She developed hepatocellular carcinoma affected by hepatitis $C$ virus-related cirrhosis and underwent posterior sectionectomy. Portal vein thrombosis developed and the portal trunk was narrowed after hepatectomy. Portal vein thrombosis resulted in high portal pressure and increased blood flow in an inferior mesenteric venous-inferior vena cava shunt, and hepatic encephalopathy with hyperammonemia was aggravated. The hepatic encephalopathy aggravated by portal vein thrombosis was successfully treated by balloon-occluded retrograde transvenous obliteration via a right transjugular venous approach without the development of other collateral vessels.
\end{abstract}

\section{Introduction}

A porto-systemic shunt often causes hepatic encephalopathy with high systemic blood ammonia in cirrhotic patients $[1,2]$. Medical therapies, such as treatment with lactulose and oral branched-chain amino acid supplementation, have a limited effect in patients with porto-systemic shunt [2]. Obliteration of the porto-systemic shunt is one possible 
treatment for porto-systemic shunt encephalopathy, and balloon-occluded retrograde transvenous obliteration (B-RTO) is an effective treatment for porto-systemic shunt encephalopathy [3-5] in addition to its application for the treatment of gastric varices [6].

Obliteration of porto-systemic shunt by B-RTO provides portal blood flow and transiently increases portal pressure $[3,7]$. Providing patients with the capacity to buffer high portal pressure and increased hepatic blood flow allows recovery of their liver function including hepatic encephalopathy $[3,8]$. However, patients who cannot buffer high portal pressure and increased portal blood flow are decompressed by the development of alternate collaterals such as esophageal varices or other collaterals [7], increasing the risk of bleeding and/or hepatic encephalopathy and ascites. Therefore, B-RTO for patients with portal trunk obstruction caused by thrombosis or tumor thrombosis has difficulties.

This report presents a case of hepatic encephalopathy aggravated by portal vein thrombosis (PVT) successfully treated by B-RTO via a transjugular vein approach.

\section{Case Report}

A 78-year-old female with hepatitis $\mathrm{C}$ virus-associated liver cirrhosis developed hepatocellular carcinoma and underwent posterior sectionectomy. The patient was admitted to the hospital 2 months later with hepatic encephalopathy (stage 1-2 by West Haven criteria) and a high systemic blood ammonia level $(246 \mu \mathrm{g} / \mathrm{dl}$ ). Liver function was Child-Pugh B (total bilirubin $1.7 \mathrm{mg} / \mathrm{dl}$, albumin $2.5 \mathrm{~g} / \mathrm{dl}$, prothrombin time $68 \%$, with mild ascites). In addition, she had extreme edema in both lower extremities. Multi detector-row computed tomography (MD-CT) and three-dimensional angiography by MD-CT revealed a porto-systemic shunt from the inferior mesenteric vein (IMV) draining to the

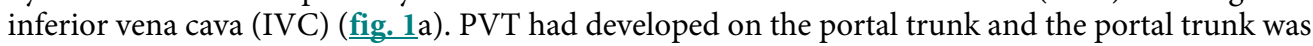
narrowed.

Anticoagulation therapy was initiated with heparin (10,000 U/day intravenously) followed by warfarin. The dosages of warfarin were adjusted to achieve an international normalized ratio of approximately 2.0 [9]. However, the PVT did not improve and the hepatic encephalopathy persisted. The resistance to anticoagulation therapy was thought to be caused by decreased portal venous blood flow accompanied by increased blood flow of IMV-IVC shunt aggravated by PVT. Therefore, B-RTO was performed to obliterate the IMV-IVC shunt via the right transjugular vein approach. B-RTO was performed as described previously [6]. A balloon catheter for B-RTO (6.5 French; Create Medic, Tokyo, Japan) was introduced into the IMV-IVC shunt via the right transjugular vein and the shunt was visualized using iopamidol. The IMV-IVC shunt was confirmed to be the only route, and the draining blood flow was blocked by inflating the balloon. $40 \mathrm{ml}$ of $50 \%$ glucose and $2 \mathrm{ml}$ of absolute ethanol were injected, and $15 \mathrm{ml}$ of $5 \%$ ethanolamine oleate with iopamidol was injected slowly in a retrograde manner (fig. 1b). Simultaneously, 4,000 U of haptoglobin (Yoshitomi, Osaka, Japan) was administered intravenously for protection against renal damage by the ethanolamine oleate sclerosant [10]. The balloon was deflated $24 \mathrm{~h}$ after the injection of the sclerosant.

The clinical symptoms of hepatic encephalopathy completely disappeared (stage 0 by West Haven criteria) 2 weeks after the treatment, and the serum ammonia level decreased immediately (fig. $2 a$ ). Serum total bilirubin decreased and albumin increased after the treatment (fig. $2 b, c)$. These results were sustained over a 5-month follow-up. Contrast-enhanced CT scans performed 2 weeks after B-RTO revealed that the narrowed portal trunk and the left branch was expanded (fig. $3 \mathrm{a}, \mathrm{b}$ ), accompanied by embolization of the IMV-IVC shunt (fig. 3c). In addition, the edema in both lower extremities and ascites disappeared after treatment. Contrast-enhanced CT and upper gastrointestinal endoscopy revealed no evidence of esophageal varices and/or gastric varices or other collateral vessels. Anticoagulation therapy with warfarin was continued at dosages of warfarin adjusted to achieve an international normalized ratio of approximately 2.0. 


\section{Discussion}

B-RTO is an effective treatment for gastric varices [6] and porto-systemic shunt encephalopathy $[3,8]$ associated with portal hypertension. Various collateral pathways of porto-systemic shunts contribute to hepatic encephalopathy, and the frequency of collateral pathways associated with the IMV is about 13\% [11]. The drainage veins of these shunts include: direct shunt to the IVC, left gonadal vein, left internal iliac vein, and right gonadal vein. B-RTO is usually performed through the femoral vein. However, other approaches or other treatment modality, such as surgical ligation, must be considered when the porto-systemic shunt in not accessible from the femoral vein. A right transjugular venous approach enabled safe and effective obliteration of the IMV-IVC shunt in the current patient. Only in a few cases was the transjugular vein approach used for B-RTO [12].

PVT is not an uncommon complication in patients with liver cirrhosis, and its incidence ranges from 2 to $26 \%[9,13]$. Abdominal surgery, such as splenectomy, is associated with the development of PVT [9], however few patients develop PVT after hepatectomy $[14,15]$. Portal pressure increases with PVT and alternate collaterals such as esophageal varices or other collaterals [7] develop to decompress the high portal pressure, which results in decreased portal flow. Since decrease in portal flow is one of the predisposing factors for PVT, patients may present resistance to anticoagulation therapy, as observed in the current patient.

Obliteration of a porto-systemic shunt by B-RTO transiently increases portal blood flow and portal pressure $[3,7]$. Liver function, including hepatic encephalopathy, is often recovered when the patients have the capacity to buffer high portal pressure and increased hepatic blood flow $[3,8]$. Therefore, B-RTO was applied to obliterate the porto-systemic shunt in the current patient to treat hepatic encephalopathy and to increase portal blood flow, followed by immediate anticoagulation therapy. The hepatic encephalopathy disappeared as expected and the narrowed portal flow expanded despite the development of PVT. In addition, the serum albumin level increased and the edema of both lower extremities and ascites disappeared. Those results occurred as a result of increased portal blood flow. Furthermore, other no collateral vessels or esophageal varices developed. However, PVT persisted, requiring anticoagulation therapy. Therefore, patients aggravated high porto-systemic shunt blood flow with acute PVT may be immediately treated with B-RTO.

This report presented a case of hepatic encephalopathy aggravated by PVT who was successfully treated by B-RTO via a transjugular venous approach. 


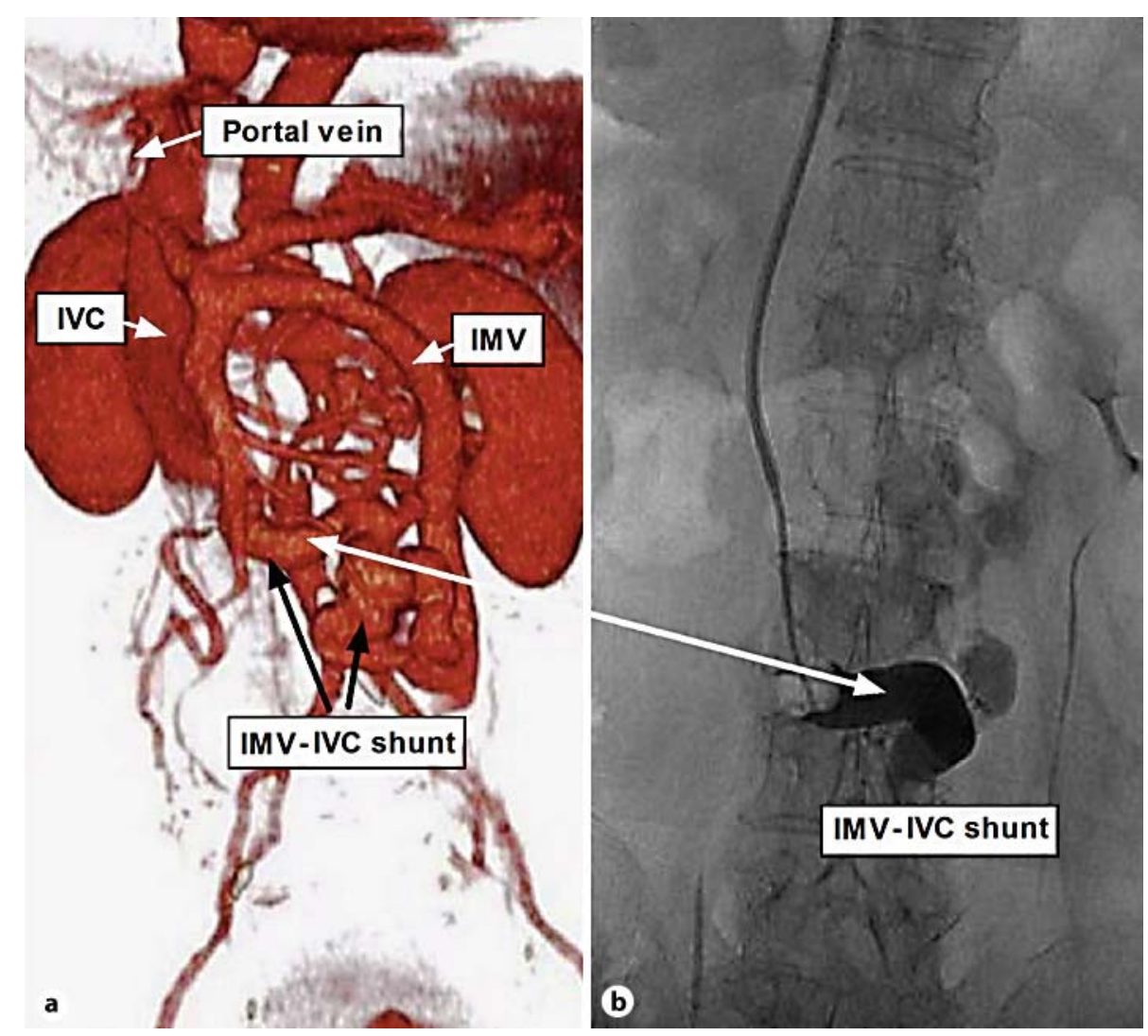

Fig. 1. MD-CT revealed a porto-systemic shunt from the inferior mesenteric vein (IMV) draining to the inferior vena cava (IVC) with narrowed portal trunk (a). The draining blood flow of the IMV-IVC shunt was blocked by inflating the balloon, and $15 \mathrm{ml}$ of $5 \%$ ethanolamine oleate with iopamidol was injected slowly in a retrograde manner $(\mathbf{b})$. 

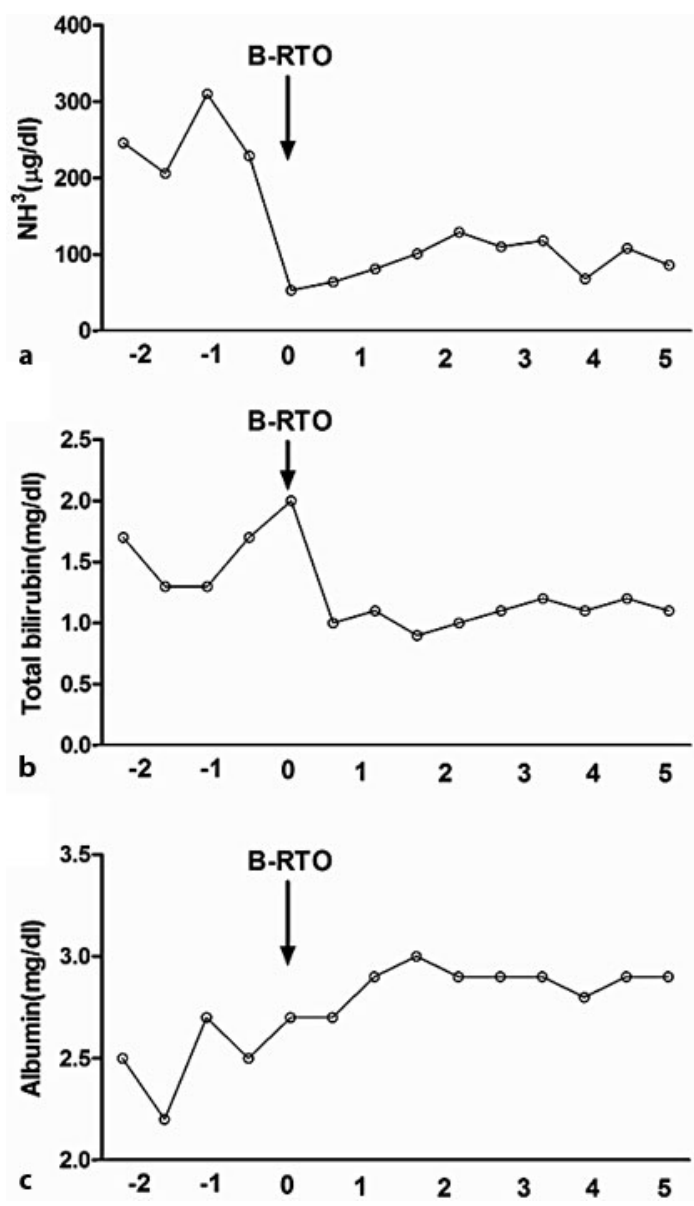

Fig. 2. The serum ammonia level decreased immediately after B-RTO (a). Serum total bilirubin level decreased and albumin level increased after the treatment $(\mathbf{b}, \mathbf{c})$. These results were sustained over a 5-month follow-up.

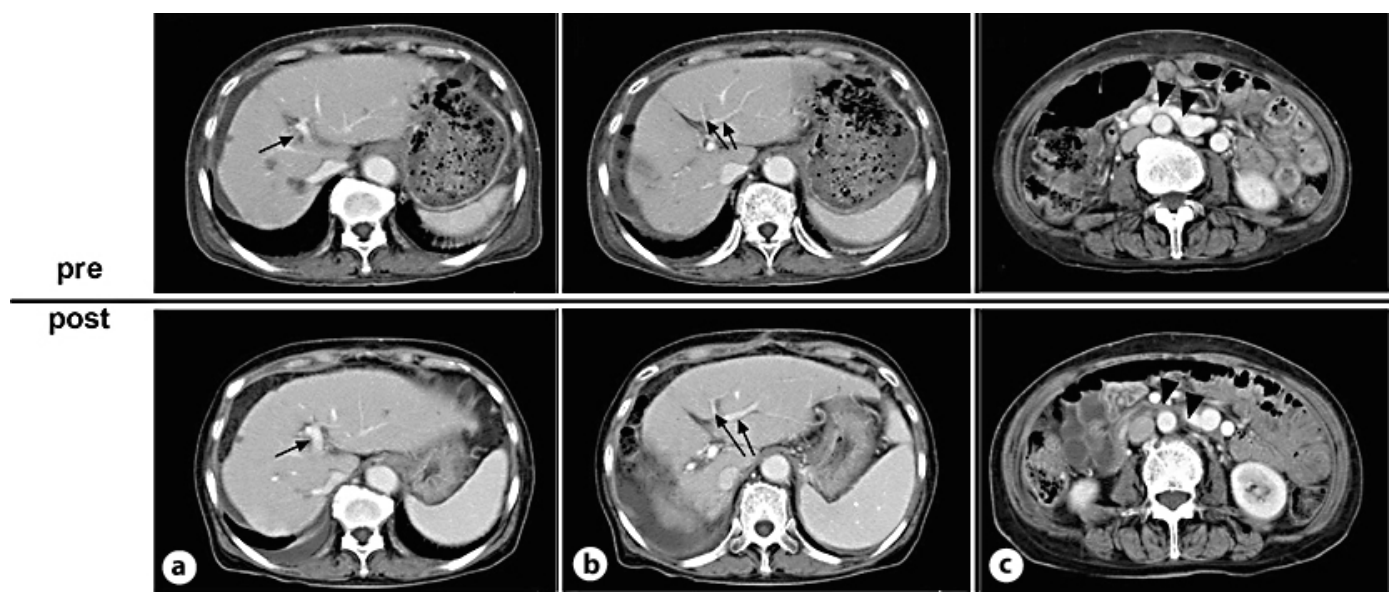

Fig. 3. Contrast-enhanced CT scans performed 2 weeks after the B-RTO revealed that the narrowed portal trunk and the left branch were expanded $(\mathbf{a}, \mathbf{b})$, accompanied by embolization of the IMV-IVC shunt $(\mathbf{c})$. 


\begin{tabular}{|c|c|c|c|}
\hline $\begin{array}{r}\text { Case Reports in } \\
\text { Gastroenterology }\end{array}$ & $\begin{array}{l}\text { Case Rep Gastroenterol 2011;5:366-371 } \\
\text { DOI: } 10.1159 / 000330287\end{array}$ & $\begin{array}{l}\text { Published online: } \\
\text { July } 8,2011\end{array}$ & $\begin{array}{l}\text { @ } 2011 \text { S. Karger AG, Basel } \\
\text { ISSN 1662-0631 } \\
\text { www.karger.com/crg }\end{array}$ \\
\hline
\end{tabular}

\section{References}

1 Bustamante J, Rimola A, Ventura PJ, et al: Prognostic significance of hepatic encephalopathy in patients with cirrhosis. J Hepatol 1999;30:890-895.

2 Yoshida T, Muto Y, Moriwaki H, et al: Effect of long-term oral supplementation with branched-chain amino acid granules on the prognosis of liver cirrhosis. Gastroenterol Jpn 1989;24:692-698.

-3 Kumamoto M, Toyonaga A, Inoue $\mathrm{H}$, et al: Long-term results of balloon-occluded retrograde transvenous obliteration for gastric fundal varices: hepatic deterioration links to portosystemic shunt syndrome. J Gastroenterol Hepatol 2010;25:1129-1135.

4 Kato T, Uematsu T, Nishigaki Y, et al: Therapeutic effect of balloon-occluded retrograde transvenous obliteration on portal-systemic encephalopathy in patients with liver cirrhosis. Intern Med 2001;40:688-691.

5 Shimizu K, Nakashima M, Fujimoto M, et al: A case of portal-systemic shunt-through inferior mesenteric vein and right internal iliac vein-encephalopathy cured by balloon-occluded retrograde transvenous obliteration (in Japanese). Nippon Shokakibyo Gakkai Zasshi 2009;106:1764-1769.

6 Akahoshi T, Hashizume M, Tomikawa M, et al: Long-term results of balloon-occluded retrograde transvenous obliteration for gastric variceal bleeding and risky gastric varices: a 10-year experience. J Gastroenterol Hepatol 2008;23:1702-1709.

7 Matsumoto A, Hamamoto N, Nomura T, et al: Balloon-occluded retrograde transvenous obliteration of high risk gastric fundal varices. Am J Gastroenterol 1999;94:643-649.

8 Miyamoto Y, Oho K, Kumamoto M, et al: Balloon-occluded retrograde transvenous obliteration improves liver function in patients with cirrhosis and portal hypertension. J Gastroenterol Hepatol 2003;18:934-942.

\9 Kawanaka H, Akahoshi T, Kinjo N, et al: Impact of antithrombin III concentrates on portal vein thrombosis after splenectomy in patients with liver cirrhosis and hypersplenism. Ann Surg 2010;251:76-83.

10 Hashizume M, Kitano S, Yamaga H, et al: Haptoglobin to protect against renal damage from ethanolamine oleate sclerosant. Lancet 1988;2:340-341.

11 Tanaka R, Ibukuro K, Abe S, et al: Treatment of hepatic encephalopathy due to inferior mesenteric vein/ inferior vena cava and gonadal vein shunt using dual balloon-occluded retrograde transvenous obliteration. Cardiovasc Intervent Radiol 2009;32:390-393.

12 Ohta M, Yasumori K, Saku M, et al: Successful treatment of bleeding duodenal varices by balloon-occluded retrograde transvenous obliteration: a transjugular venous approach. Surgery 1999;126:581-583.

13 Okuda K, Ohnishi K, Kimura K, et al: Incidence of portal vein thrombosis in liver cirrhosis. An angiographic study in 708 patients. Gastroenterology 1985;89:279-286.

14 Sozen H, Karakayali H, Moray G, et al: Analysis of postsurgical complications in 75 living liver transplantation donors. J Gastrointest Surg 2006;10:646-651.

15 Koh MK, Ahmad H, Watanapa P, et al: Beware the anomalous portal vein. HPB Surg 1994;7:237-239; discussion 239-240. 